\title{
Summary of the American College for Advancement in Medicine November 2004 Conference on Emerging Concepts in Immunology
}

\author{
Stephen A. Feig ${ }^{1}$, Neal Speight ${ }^{2}$, Eleanor Hynote ${ }^{3}$ and Allan Magaziner ${ }^{4}$ \\ ${ }^{1}$ Integrative Medical Consulting, 208 Vista Bella Drive, Santa Cruz, CA 95060, ${ }^{2}$ The Center for Wellness, \\ 2317 Randolph Road, Charlotte, NC 28207, ${ }^{3}$ Phoenix Wellcare, 935 Trancas St Ste 1A, Napa, CA 94558 \\ and ${ }^{4} 1907$ Greentree Road, Cherry Hill, NJ 08003, USA
}

The autumn 2004 session of the American College for Advancement in Medicine's 162nd International Educational Conference on Complementary, Alternative and Integrative Medicine (CAIM) took place November 17-21, 2004 at the Sheraton San Diego Hotel and Marina in San Diego, California, USA. This event served as testament to the overwhelming professional interest in CAIM therapies and the science supporting evidence-based CAIM therapies. Embraced by Southern California's warm sunny skies and temperate gentle breezes, over 500 physicians, health practitioners and medical professionals convened at ACAM's 'A Blast to the Future-Emerging Concepts in Immunology' conference, in order to learn about the latest science behind a wide variety of exciting new CAIM therapies.

The American College for Advancement in Medicine (ACAM) is a not-for-profit medical society dedicated to educating physicians and other health care professionals on the latest findings and emerging procedures in preventive/nutritional medicine. As the voice of CAIM, ACAM strives to increase awareness and recognition of CAIM therapies and play an active role in shaping the future of the CAIM movement.

Celebrating more than a quarter century of service, ACAM represents nearly 1000 physicians in 30 countries. ACAM is the largest and oldest organization of its kind in the world dedicated to providing its members with educational excellence in integrative medicine. ACAM's leading edge educational programming facilitates the incorporation of clinically relevant biomedical therapies by fast tracking scientific advancements in nutritional medicine, nutraceuticals and ethno-pharmacology into the practice of cutting-edge CAIM.

For reprints and all correspondence: Stephen A. Feig D. O. c/o The American College for Advancement in Medicine, 23121 Verdugo Drive, Suite 204

Lagma Hills, CA 92653, USA. Tel: +949-583-7666 Fax: +949-455-9679:

Email: info@acam.org
ACAM was the first major medical organization to educate its members about such now well accepted concepts as insulin resistance/metabolic syndrome, the detrimental effects of homocysteine, the protective effects of antioxidants, the clinical uses of essential fatty acids, cancer chemoprotection therapies with diet and herbs, testing for cardiosensitive C-reactive protein, the benefits of natural thyroid replacement and nutritional medicine as applied to psychiatry.

Scientific presentations at ACAM's autumn 2004 convocation addressed a multitude of topics relevant to physicians and scientists interested in using CAIM therapies for the evaluation and treatment for a wide variety of diseases. Presentations on advances in laboratory medicine included the laboratory assessment of complex diseases and the assessment of immune integrity through evaluation of T- and B-cell subsets and natural killer (NK) cell activity. CAIM treatments of cancer were addressed through evaluating the efficacy of herbs and immunemodulating peptide analogues in the treatment of cancer.

Colleen Hayes, PhD, Professor of Biochemistry and Medical Microbiology in the Department of Biochemistry at the University of Wisconsin-Madison and Chairperson for the Department of Defense Breast Cancer Research Program in the Cell Biology Study Section, presented a fascinating lecture on vitamin D's relationship to the endocrine system and autoimmune diseases. She provided data from an animal model of autoimmune encephalomyelitis and the pro-apoptotic influence of 1,25-dihydroxyvitamin D3 on inflammatory cells leading to their selective destruction.

Edwin Cooper, PhD, Distinguished Professor, Department of Neuroimmunology at the David Geffen School of Medicine at UCLA was presented with the Norman E. Clark Award for Innovation in Medicine for his contributions to advancing the field of CAIM and for his groundbreaking work as the Founding Editor in Chief of eCAM. Neal Speight, MD,

(C) The Author (2005). Published by Oxford University Press. All rights reserved.

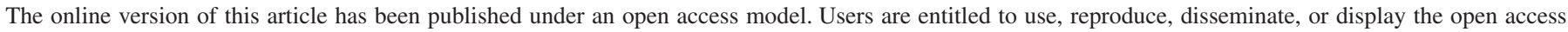

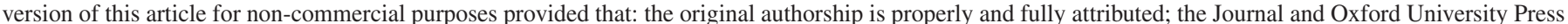

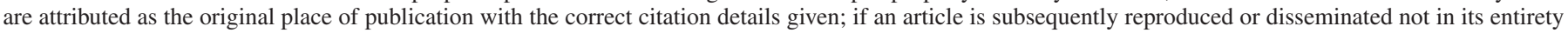
but only in part or as a derivative work this must be clearly indicated. For commercial re-use, please contact journals.permissions@ oupjournals.org 
ACAM's conference co-chairman, gave Dr Cooper this award. Dr Cooper also gave a keynote lecture on Emerging Concepts in Immunology, where he reviewed the history of immunology and its interface with various CAIM therapies.

A complete list of conference presentations can be found at the end of this article.

In addition to the many exciting presentation at the scientific conference, ACAM added a number of new and updated preconference workshops to their quality line up of educational programs. A highly popular 2-day mesotherapy workshop, Chaired by ACAM's President Dr Allan Magaziner, highlighted ACAM's pre-conference workshops. Mesotherapy was developed originally in France by Dr Michel Pistor and is currently being administered by over 15000 doctors in France. Mesotherapy involves the injection of vitamins, minerals, amino acids, homeopathics and other biologicals, and can help with body sculpting, weight loss, cellulite reduction, wrinkle reduction and facial rejuvenation. ACAM is the first medical organization to teach a combination of classical mesotherapy, bio-mesotherapy and lipodissolve at one conference.

Other new and updated workshops included 'Integrating Nutrition into Medical Practice' a 1-day workshop on integrating the fundamentals of nutrition into complementary and alternative medicine clinical practice, and 'Translating CAIM into Practice: A Management/Legal Workshop'.

ACAM's President, Dr Allan Magaziner commented, 'I'm proud to say that our Fall 2004 ACAM convention in San Diego was one of our best ever. There was a feeling of renewed excitement and energy that was experienced by hundreds of attendees and exhibitors. Participants were thrilled with the professionalism, depth of cutting-edge knowledge and stellar presentations that were shared by our scientific and clinical speakers.'

ACAM is a professional medical organization for physicians interested in learning about evidence-based CAIM therapies. ACAM's goals are to improve skills, knowledge and diagnostic procedures as they relate to complementary and alternative medicine; to support research; and to develop awareness of alternative methods of medical treatment. ACAM's next scientific conference 'Menopause, Andropause: Power in Transition' will be held at Disney's Coronado Spring Resort in Orlando, Florida, May 18-22, 2005. Workshop topics include Esthetic Mesotherapy, Heavy Metals and Chelation Therapy and an in-depth Nutrition Certification Seminar. For further information, please call ACAM at 800-532-3688 (in the USA) or 949-583-7666 (outside the USA) or register on line at http://www.acam.org

\section{Summary of Presentations}

\section{Ari Vojdani PhD, MT}

CEO of Immunosciences Labs, and Assistant Researcher at the Department of Neurobiology at UCLA: "Laboratory of Immunology for the Assessment of Complex Diseases".

With more than 100 peer-reviewed articles and 20 patents to his credit, Dr Vojdani reviewed the key role infections and xenobiotics play in altering immune function and how they lead to the development of complex disease states. The following concepts were reviewed: (i) the cross-reactivity of tissues and cellular antigens, including myelin basic protein- specific $\mathrm{T}$ cells, with multiple microbial peptides; (ii) how haptenic chemicals such as mercury can bind to different tissue antigens and induce allergic and autoimmune reactions; (iii) the prototypic Th2 autoimmunity induced by mercury and its dependence on interferon (IFN)- $\gamma$ and other proinflammatory cytokines in animal models and in children with autism; and (iv) the practical assessment of the immune system in the setting of complex disease states utilizing testing for: T-cell helper/suppressor ratios, antinuclear antibody (ANA), fibrillarin, chromatin, mercury, neurofilament, tubulin and antibodies to neuron-specific antigens. These and many other immunological tests were reviewed for early diagnosis of autoimmune and neuroimmune diseases induced by infections and toxic chemicals. Celiac, Crohn's disease, ulcerative colitis, myocarditis, lupus, multiple sclerosis and Lyme disease were a few examples of the diseases, which were discussed.

\section{Mary Hardy, MD}

A complementary and alternative medicine expert for research projects conducted by the Southern California Evidence-based Practice Center at the Land Corporation.

\section{Herbs and Cancer}

Dr Hardy reviewed the use of medicinal plants as chemopreventative agents, the mechanisms by which plants can affect the genesis of cancer, and the potential interaction between medications and chemotherapy.

Dr Hardy reviewed the following concepts: (i) characteristics of cancer and the stepwise nature of cancer development with a major emphasis on mutations and proliferation; (ii) different opportunities for intervention using herbal therapies which address different cellular and molecular targets involved in the development of cancer; (iii) examples of natural chemopreventative agents such as green tea, panax ginseng, turmeric, soy, medicinal mushrooms and their molecular mechanisms; and (iv) immune enhancement properties of these agents, in particular, medicinal mushrooms, on cell-mediated immunity.

\section{Jesse Stoff, MD}

Medical Doctor, Certified Naturopathic Physician, Acupuncturist and Homeopathic Physician. Graduate of New York Medical College and Fellow of Royal London Homeopathic Hospital in London, UK.

\section{Immune Reconstitution in the Face of Severe or Chronic Disease}

Dr Stoff's presentation focused primarily on the assessment of immune integrity through evaluation of T- and B-cell subsets, and NK cell activity. He reviewed the patterns of immune dysfunction seen in various viral states and suggested therapies designed to restore proper balance to the immune system damaged by viral infections or heavy metal exposure. 


\section{Rashid Buttar, DO}

Visiting Scientist at North Carolina State University. Director of the Clinical Research and Development at V-SAB Medical Labs, Inc. Medical Director of Advanced Concepts in Medicine.

\section{Step Non-traditional Medical Approach in the Treatment of Cancer Utilizing Immune Modulating Peptide Analogs}

Dr Buttar's lecture summarized the importance of preventive approaches to cancer and emphasized that optimizing nutritional and environmental balance prior to onset of cancer is the most practical approach. He reviewed the common characteristics of most cancers, the disruption of nutritional status and toxic body burden that may contribute to oncogenesis, the importance of systemic optimization of the cancer patient, and monitoring biological markers to assess efficacy of treatment. He reviewed his peptide modulators and their potential benefit in the management of cancer and also emphasized the potential role of heavy metals in many cancers. Participants were very interested in the structure of this peptide, which was not discussed during Dr Buttar's presentation.

\section{Farrand Robson, DDS}

Member of the Washington State Dental Association, the ADA, and the Academy of Head, Neck, and Facial Pain, A Fellow of The International College of Cranio-mandibular Orthopedics, and a Diplomat of The American Academy of Pain.

\section{Systemic Implications of Oral Functions}

Dr Robson reviewed the role of craniofacial anatomic dysfunction and its influence on the autonomic nervous system leading to commonly seen, but often mismanaged, illnesses such as chronic headaches, upper back pain and fibromyalgia. He emphasized the importance of proper occlusion, and how malocclusion can frequently lead to systemic illness that often manifests as sleep disordered breathing (SDB) and TMJ syndrome with its related problems. Finally, he provided a practical solution to these problems utilizing a unique dental prosthesis that is now clinically available.

\section{Colleen Hayes, PhD}

Professor of Biochemistry and Medical Microbiology in the Department of Biochemistry at the University of WisconsinMadison. Member of the American Association of Immunologist, the American Association for the Advancement of Science, and the American Institute of Nutrition. Chairperson for the Department of Defense Breast Cancer Research Program in the Cell Biology Study Section.

\section{The Vitamin D Endocrine System and Autoimmune Disease}

Dr Hayes reviewed vitamin D3 biosynthesis, its conversion to the steroid hormone 1,25-dihydroxyvitamin D3 and the role this hormone has in the prevention of autoimmune diseases such as multiple sclerosis, diabetes, inflammatory bowel disease and arthritis. She showed how exacerbations of multiple sclerosis seem to follow the lack of seasonal sunlight in the more northern latitudes and its correlation to lower vitamin D levels. The same trend was observed in insulin-dependent diabetes mellitus and irritable bowel syndrome. She then provided data from an animal model of autoimmune encephalomyelitis and the pro-apoptotic influence of 1,25-dihydroxyvitamin D3 on inflammatory cells leading to their selective destruction.

\section{Mark Estes, PhD}

Professor and Senior Scientist in the Department of Pediatrics, Microbiology, and Immunology, and Pathology at the Sealy Center for Vaccine Development at the University of Texas Medical Branch.

\section{Alternation in Immune Responses to Bacterial Infection in Mice Fed Dietary Phytoestrogen Genistein}

Dr Estes' area of research focused on the role of sex hormones in modulating immune function: specifically the polarizing effect of genestein on the Th1 versus Th2 response in the face of infection with $M$. avium. His studies demonstrated that ER- $\alpha$ (estrogen eeceptor- $\alpha$ ) signaling and dietary genistein inhibited production of type 1 cytokines (IFN- $\gamma$ and interleukin-18) in response to chronic M. avium infection in female mice. IFN- $\gamma$ plays an important role in controlling intracellular bacterial infection and is mainly produced by $\mathrm{T}$ cells and NK cells. In his research, comparison of cytokine production in immunocompetent versus immunodeficient mice indicates that in the setting of chronic infection with $M$. avium, the effects of ER- $\alpha$ and genistein on cytokine production are probably mediated via signaling in $\mathrm{T}$ cells.

\section{Carolyn McMakin, MA, DC}

Clinical Director of the Fibromyalgia and Myofascial Pain Clinic of Portland, Oregon.

\section{Modulation of Inflammatory Cytokines Using Frequency Specific Therapy}

Dr McMakin reviewed the use of biological resonance (FSM; frequency-specific microcurrent) as it applies to the reduction of inflammation in a mouse model and in the treatment of fibromyalgia associated with cervical trauma. She reviewed unpublished animal data showing significant reductions in lipoxygenase- and cyclooxygenase-mediated inflammation. Specifically, in a mouse model, the ears were painted with arachidonic acid in ethanol leading to dramatic edema that could be reduced by $70 \%$ within 4 min with the use of FSM. The rationale behind such powerful reduction in inflammation was reviewed.

\section{Hugh Fudenberg, MD, PhD}

Chief Editor of the Journal of Clinical Immunology and Immunopathology

\section{Hazards of Vaccines and of the Thimerosol in them}

Dr Fudenberg reviewed the evidence suggesting vaccines, specifically measles vaccine, may be contributing to 
developmentally delayed disorders, such as ADHD and autism. He cited references supporting high levels of antibodies in the spinal fluid of patients with infantile onset autism.

\section{Edwin Cooper, PhD}

Professor, Department of Neuroimmunology David Geffen School of Medicine at UCLA.

\section{Emerging Concepts in Immunology}

Dr Cooper gave the keynote lecture on the field of immunology. Reviewing its history and evolution, he summarized the role of inflammation in the ancient literature to the present day. He reviewed research in the field of complementary and alternative medicine as it relates to immune tolerance and stimulation, showing the vast number of compounds being evaluated for their potential medicinal value. He specifically reviewed the enzyme lumbrokinase (an anticoagulant extracted from earthworms) and its benefit in the treatment of hypercoagulable states.

\section{Shari Lieberman, PhD}

Certified Nutrition Specialist, Fellow of the American College of Nutrition, Member of the New York Academy of Science, Author or contributing editor to many books including the AMA's 5th Edition of Drug Evaluations.

\section{Nutriceuticals in the Battle Against Cancer}

Dr Leiberman reviewed the role of catechins, specifically EGCG (epigallocatechin-3-gallate), as an inhibitor of metastasis by blocking matrix metalloproteinase, by specific induction of cancer cell apoptosis and as a tyrosine kinase receptor inhibitor. The results of studies showing its benefit in cervicitis, mild to moderate cervical dysplasia and HTLV-1 were reviewed. Data on curcumin, lycopene and ascorbic acid in cancer patients and in vitro studies were also covered.

\section{Richard Neubauer, MD}

Medical Director of Ocean Hyperbaric Neurologic Center. Medical Graduate, University of Virginia with honors. Pioneer of several emerging applications of hyperbaric oxygen in the treatment of stroke.

\section{Hyperbaric Oxygenation in Mitochondrial Cytopathies}

Dr Neubauer reviewed the history of hyperbaric oxygenation (HBO), the effect of pressurized oxygen in acute brain insult, its effect in chronic brain insult, the mitochondrial electron transport chain and the potential influence of $\mathrm{HBO}$ on gene transcription.

\section{Mohamed Rafi, PhD}

Assistant Professor in the Department of Food Science at the Cook College, Rutgers, University. Postdoctoral Fellow and Research Teaching Specialist at the Cancer Institute of New
Jersey. Recipient of the Gallo Award for Outstanding Cancer Research

\section{A Novel Bcl-2 Phosphorylating Molecule in Licorice: an Ultimate Answer to Cancer?}

Overexpression of Bcl-2 prevents the release of cytochrome $\mathrm{c}$ from the mitochondria, a critical event in the apoptotic pathway. Bcl-2 in higher concentrations protects cells from deathpromoting stimuli, whereas its underexpression increases a cell's sensitivity to death. Dr Rafi reviewed his original research on the licorice-derived polyphenol, $\beta$-hydroxy DHP (a component of PC-SPES) showing how it induces apoptosis, $\mathrm{G}_{2} / \mathrm{M}$ cell cycle arrest, Bcl-2 phosphorylation and altered microtubule structure.

\section{Michael Zeligs, MD}

Founder and CEO of BioResponse Nutrients currently collaborating with the Chemoprevention Branch of the NIH on the benefit of DIM in estrogen metabolism. Masters Degree on Stress-Physiology from UC, Santa Barbara. MD Degree from UC-Irvine.

\section{Reversing Estrogen Dominance: Intervention with Cruciferous Diindolylmethane (DIM)}

Dr Zeligs showed how 4-hydroxy estrogen contributes to estrogen-related disorders including breast cancer risk. He then showed studies on the role of diindolylmethane (DIM) in increasing 2-hydroxy estrogen, which is associated with specific health benefits including reducing cancer risk and offering protection from human papilloma virus (HPV). DIM is a potent antioxidant and normalizes estrogen metabolism. Dr Zeligs presented studies showing reduced breast and prostate cancer growth in animals.

\section{Richard Hansen, DMD}

Dental Private Practice. Faculty member at UCLA's Center for Aesthetic Dentistry. Advisory Board Member: American Preventive Medical Association.

\section{The Oral Immune System}

The oral immune system is composed of the mucosal barrier system, which creates a large output of secretory $\operatorname{IgA}(\mathrm{SIgA})$. If immunity is low, $\operatorname{SIgA}$ output is reduced and infections enter more easily through the mucosal barrier into the blood. Oral infections are related to heart disease, bacterial endocarditis, stroke, diabetes and vascular inflammation. Dr Hansen outlined the hazards of dental materials and procedures on the oral cavity. Harmful dental materials include mercury, cadmium, nickel and plastics. Root canals can cause chronic low-grade infections and chronic inflammation. TMJ and bite stress can also force breakdown of bone and immune membranes, which can also lead to oral infection. 


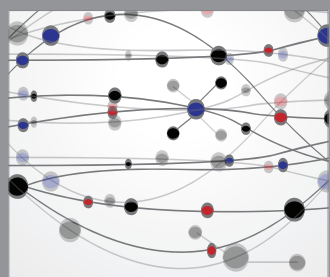

The Scientific World Journal
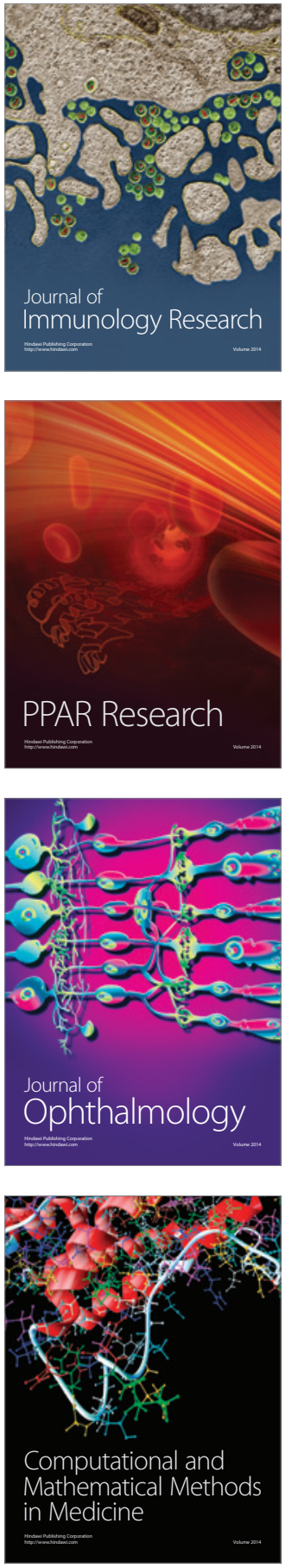

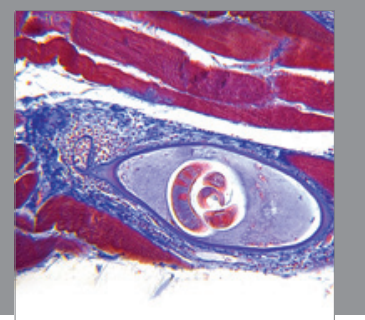

Gastroenterology

Research and Practice
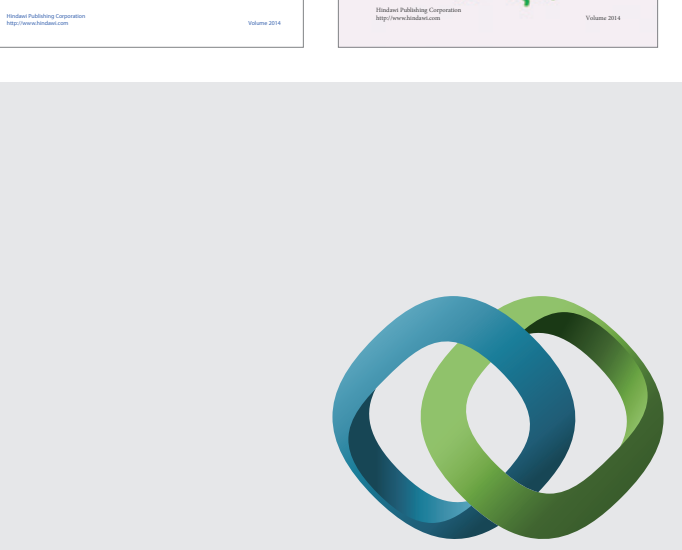

\section{Hindawi}

Submit your manuscripts at

http://www.hindawi.com
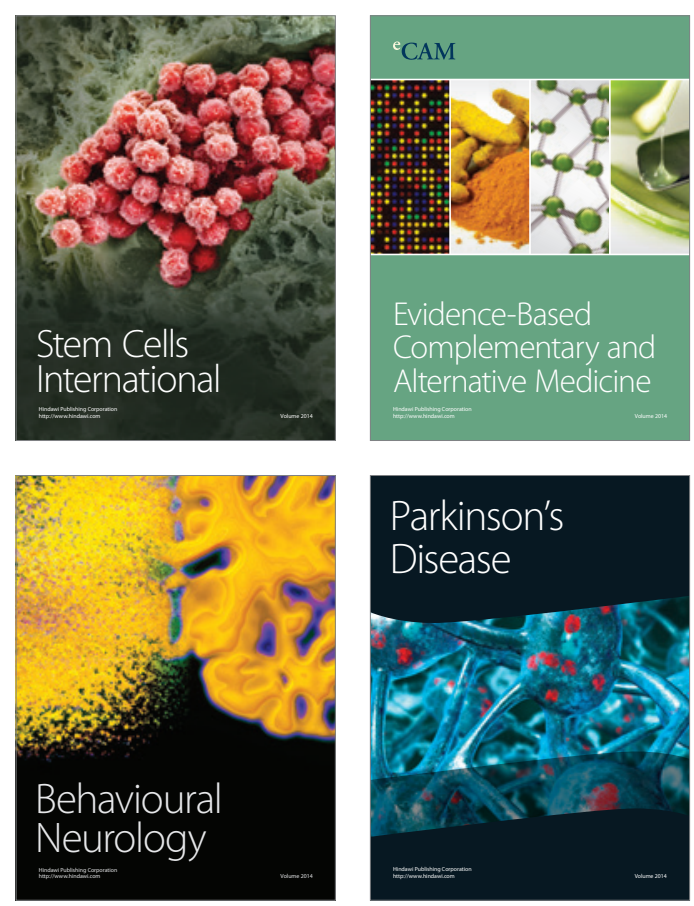

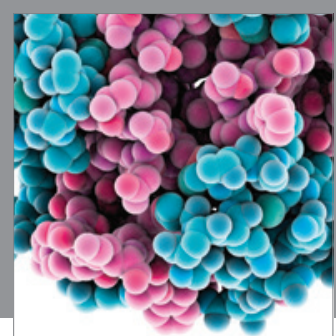

Journal of
Diabetes Research

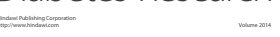

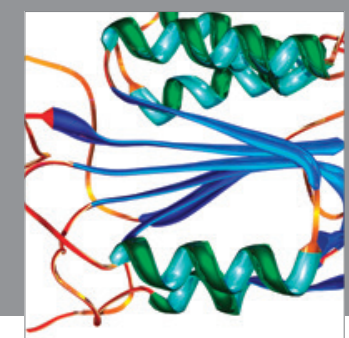

Disease Markers
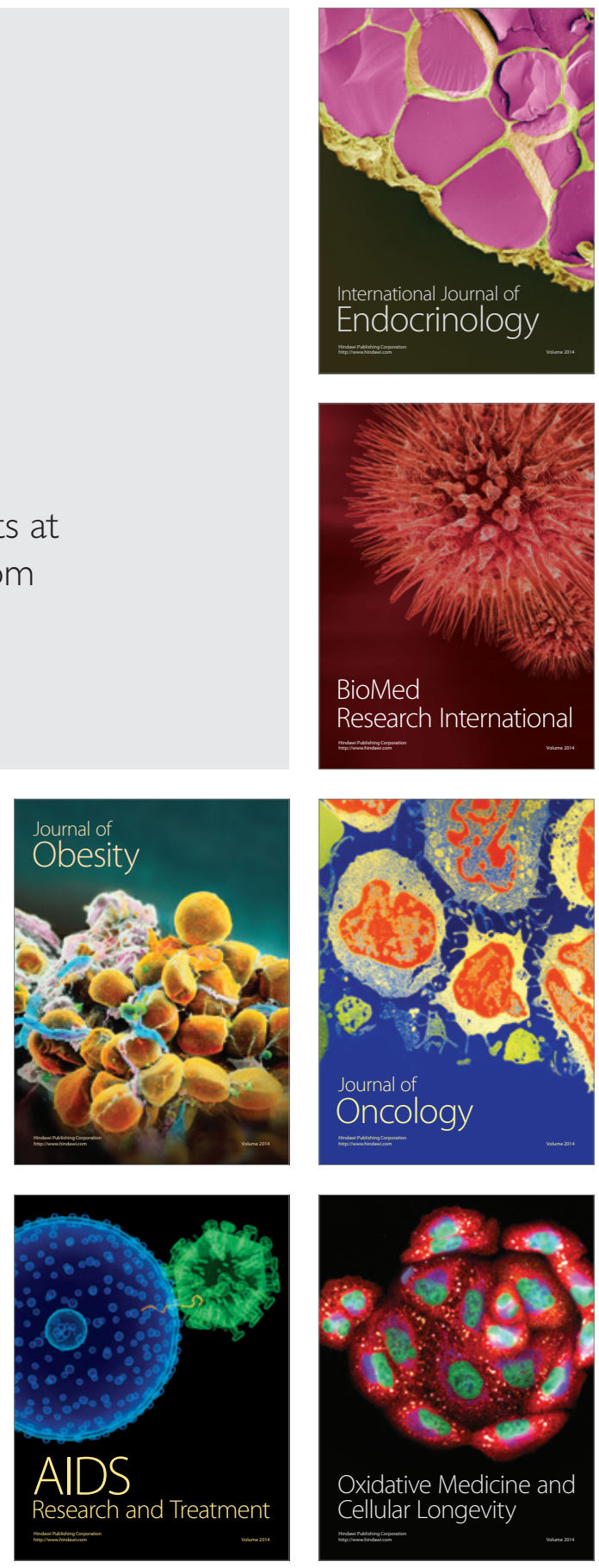